\title{
La percepción de control de la ansiedad en el trastorno límite de la personalidad
}

\author{
Minerva Romero Clausell \\ al225785@uji.es \\ MARÍA VICENTA NAVARRO \\ JORGE OSMA \\ osma@unizar.es \\ AZUCENA García-Palacios \\ azucena@uji.es
}

\section{Resumen}

Introducción: El trastorno de personalidad límite (TLP) se caracteriza por un patrón de inestabilidad en las relaciones interpersonales, autoimagen, afecto, problemas en el control de la ira e impulsividad. La mayoría de estudios sobre las vulnerabilidades emocionales en el TLP se han centrado en el rol de la impulsividad-agresión, la inestabilidad afectiva y, más generalmente, sobre la vulnerabilidad emocional; sin embargo, no se ha prestado demasiada atención al papel de la ansiedad. La literatura muestra que existen algunos estudios sobre la ansiedad y también sobre la sensibilidad a la ansiedad en el TLP, pero se conoce muy poco sobre la influencia de la percepción de control de la ansiedad (PCA). Existe evidencia empírica sobre la influencia de la PCA en los modelos de trastorno de pánico (TP); por ello, dada la relevancia que la regulación emocional tiene en la etiología y mantenimiento del TLP, y la gran comorbilidad que existe entre el TLP y los trastornos de ansiedad, el presente estudio tiene como objetivo comparar los niveles de PCA entre el TLP y el TP. Metodología: Para medir el nivel de PCA administramos el inventario de control de la ansiedad (ACQ-R) a dos muestras distintas, una de pacientes con TLP $(\mathrm{N}=53)$ y otra de pacientes con TP $(\mathrm{N}=52)$. $\operatorname{Re}$ sultados: Los resultados indican diferencias estadísticamente significativas en dos subescalas del ACQ-R (control de la amenaza y control del estrés) y en el ACQ-R total. Discusión: Los pacientes con TLP presentan una peor regulación de la ansiedad que los pacientes con TP.

Palabras clave: trastorno de personalidad límite, trastorno de pánico, ansiedad, percepción de control de la ansiedad, ACQ-R.

\section{Abstract}

Introduction: Borderline personality disorder (BPD) is characterized by a pattern of instability in interpersonal relationships, self-image, affection, anger control and impulsivity. Most studies on the emotional vulnerabilities in BPD have focused on the role of 
impulsivity-aggression, affective instability, and more generally about emotional vulnerability. However, the role of anxiety has not been studied in deep. Literature shows that there are some studies on anxiety and on anxiety sensitivity in BPD, but very little is known about the influence of perceived anxiety control (PAC). There is empirical evidence about the influence of PAC in models of panic disorder (PD). Therefore, considering the importance that emotional regulation has in the etiology and maintenance of PD, and the high comorbidity that exists between BPD and anxiety disorders, the aim of this research is to compare the levels of PCA between BPD and PD. Method: To measure the level of PCA, we used the Anxiety Control Questionnaire (ACQ-R) in two different samples of patients, BPD $(N=53)$ and PD $(N=52)$. Results: The results indicate statistically significant differences in two subscales of the ACQ (Anxiety Control Questionnaire) (control of the threat and control of stress) and in total ACQ. Discussion: BPD patients present a worse regulation of anxiety than PD patients.

Keywords: borderline personality disorder, panic disorder, anxiety, perceived anxiety control, ACQ-R.

\section{Introducción}

El trastorno de personalidad límite (TLP) se caracteriza por un patrón persistente de inestabilidad en las relaciones interpersonales, autoimagen, afecto, problemas en el control de la ira e impulsividad (APA, 1994).

Se ha observado que los individuos con TLP comparten ciertas características en el contexto de desarrollo, lo que Linehan (1993) denominó ambiente invalidante. En el ambiente invalidante la experiencia emocional es desacreditada e invalidada independientemente de la validez real de la misma, por lo que los individuos no aprenden a etiquetar las emociones, a modular la expresión emocional o a tolerar el malestar. La combinación de un ambiente invalidante junto con una vulnerabilidad biológica a la experiencia emocional provoca que el individuo desarrolle una elevada sensibilidad a los estímulos emocionales, realice respuestas emocionales extremas y requiera de más tiempo de lo habitual para regresar a su estado anímico basal. Los individuos con TLP desarrollan una desregulación emocional que resulta en una subsecuente desregulación comportamental, es decir, llevan a cabo comportamientos difíciles de controlar que provocan un deterioro en el funcionamiento de la persona afectada. Aunque estos comportamientos produzcan alivio momentáneo, interactúan en un círculo vicioso donde el malestar de las consecuencias provoca altos niveles de rumiación, por lo que aumenta la intensidad del afecto negativo y, consecuentemente, la intensidad de respuesta emocional y así seguidamente (Crowell, Beauchaine y Linehan, 2009).

La mayoría de estudios sobre las vulnerabilidades emocionales en el TLP se han centrado en el rol de la impulsividad-agresión (Siever y Davis, 1991; Skodol y cols., 2002), la inestabilidad afectiva (Siever y Davis, 1991; Siever y cols., 2002; Skodol y cols., 2002) y, más generalmente, sobre la vulnerabilidad emocional (Linehan, 1993; Livesley y cols., 1998; Zanarini y Frankenburg, 1997); sin embargo, no se ha prestado demasiada atención al papel que desencadena la ansiedad (Gratz y cols., 2008).

Existe evidencia empírica de que los pacientes con TLP muestran síntomas elevados de ansiedad (Gunderson y Singer, 1975; Snyder y Pitts, 1988) y también observamos una elevada comorbilidad con trastornos de ansiedad (McGlashan y cols., 2000; Zimmerman y Mattia, 
1999; Leichsenring y cols., 2011). Así mismo, la literatura señala que los sujetos con TLP utilizan la evitación para reducir los síntomas relacionados con la ansiedad que son percibidos como amenazantes; sin embargo, estos acaban acentuándose debido a una deficiencia en la regulación emocional (característica central en los pacientes con TLP) (Linehan, 1993; Gratz y Gunderson, 2006).

Otros estudios se han centrado en la influencia de la sensibilidad a la ansiedad (SA) en el TLP, concepto que difiere del rasgo ansiedad (Taylor, Koch y Crockett, 1991; Sandín, Chorot y McNally, 2001). Barlow (2002) describe la ansiedad como un proceso cognitivo-afectivo donde el individuo tiene un sentido de impredictibilidad e incontrolabilidad de los acontecimientos y las emociones potencialmente negativos y perjudiciales. Este sentido se asocia con activación fisiológica, aprensión ansiosa e incertidumbre sobre la habilitad de manejar los miedos. Por el contrario, la SA se entiende como la creencia que la ansiedad y sus sensaciones tienen consecuencias peligrosas o dañinas por lo que desarrollan miedo a las sensaciones de ansiedad (Reiss y McNally, 1985). De esta forma, una persona puede tener un elevado rasgo de ansiedad (tendencia a experimentar ansiedad ante cualquier evento estresante), pero no experimenta miedo a los síntomas de ansiedad (Sandin y cols., 2004). Se ha observado que los pacientes con TLP tienen mayores niveles de SA comparado con los pacientes sin un trastorno de personalidad (Gratz y cols., 2008). Además, estos pacientes mostraron niveles de SA comparables con pacientes con diagnóstico de trastorno de ansiedad generalizada, trastorno obsesivo compulsivo y trastorno de ansiedad social (Taylor y cols., 1992).

Como hemos podido apreciar, existen varios estudios sobre la ansiedad y también sobre la sensibilidad a la ansiedad en el TLP; sin embargo, sabemos muy poco sobre la influencia de la percepción de control de la ansiedad (PCA) en el TLP. EI PCA se podría definir como la creencia personal de que el individuo tiene capacidad de control sobre uno mismo y sobre las amenazas o eventos externos (Mardiyono, Songwathana y Petpichetchian, 2011). Las teorías actuales sobre desarrollo y mantenimiento de los trastornos emocionales hacen especial hincapié en el control percibido (Chapman, Kertz y Woodruff-Borden, 2009). Algunos investigadores han propuesto que la percepción de que los eventos indeseables son impredecibles e incontrolables es una característica central de la ansiedad y de los trastornos del estado de ánimo, representada por las altas tasas de comorbilidad entre ellos (Barlow, 2002; Zvolensky, Lejuez y Eifert, 2000). En lo que respecta a los trastornos de ansiedad, existe evidencia empírica sobre la influencia de la percepción de control en los modelos del trastorno de pánico (Barlow, 2002; White y Barlow, 2002). De hecho, se ha observado que los individuos con TP a menudo piensan que no pueden controlar eficazmente sus experiencias emocionales o las situaciones vitales complicadas (Rapee, Craske, Brown y Barlow, 1996) y utilizan drásticos esfuerzos para prevenir o reducir temporalmente sus estados de ansiedad (Cox, Endler, Swin-son y Norton, 1992; Zvolensky, Bernstein, Marshall y Feld-ner, 2006).

Sin embargo, no conocemos cómo funciona la PCA en uno de los trastornos de personalidad más estudiados, el TLP (Ferrer, 2013). Por lo tanto, dada la relevancia que la regulación emocional tiene en la etiología y el mantenimiento del TP (Osma, Garcia-Palacios y Botella, 2014), así como ocurre en el TLP (Linehan, 2003), y la elevada comorbilidad que existe entre el TLP y los trastornos de ansiedad, consideramos que un mejor conocimiento de la asociación entre el TLP y el TP, como conocer la influencia y el efecto modulador de la PCA en ellos, nos puede ayudar a desarrollar mejores técnicas de prevención y tratamiento así como contribuir a la comprensión de los mecanismos etiológicos. Por ello, el objetivo del presente estudio será comparar los niveles de la percepción del control de la ansiedad en dos muestras distintas, una de pacientes con TLP y otra de pacientes con TP. Nuestras hipótesis son dos, el grupo de TLP y el grupo de TP tendrán niveles parecidos de PCA y los dos grupos tendrán niveles bajos de PCA. 


\section{Método}

\section{Participantes}

La muestra está compuesta por 105 participantes de los cuales 52 tienen como diagnóstico TP y 53 TLP. Los participantes con TP forman un grupo equitativo siendo 26 hombres y 26 mujeres, con una edad media de 32 años. De los 52 participantes con TP, el 76,9\% vinieron del Servicio de Asistencia Psicológica (SAP) de la Universitat Jaume I y el 21,2\% del centro clínico Creos. Respecto a los participantes con TLP, todas son mujeres y con una edad media de 26,6 años. El grupo de TLP proviene del centro clínico Previ.

\section{Medidas}

Anxiety Control Questionnaire-Revised (ACQ-R; Brown y cols., 2004). El ACQ (Rapee y cols., 1996) es un autoinforme de 30 ítems diseñado para analizar el nivel de percepción de control sobre la ansiedad y los eventos relacionados con esta (Brown, 2004). Su versión más reciente, el ACQ-R, está compuesto por 15 ítems y evalúa la percepción de control sobre las respuestas emocionales internas de ansiedad. Lo conforman tres factores: la percepción de control emocional, compuesto por 5 ítems que reflejan la habilidad que cree tener el individuo para controlar sus emociones («Soy capaz de controlar la intensidad de mi ansiedad»), la percepción de control de amenaza, formado por 6 ítems que refieren a la creencia de que los eventos amenazadores están fuera de control de uno mismo («Hay poco que pueda hacer para cambiar acontecimientos amenazantes») y, por último, la percepción de control de estrés, compuesta por 4 ítems, que reflejan la percepción de dificultad a la hora de manejar la emociones en situaciones estresantes («En momentos de estrés es probable que pierda el control»). La escala de respuesta es de 6 puntos que van desde « $0=$ totalmente en desacuerdo» a «5 = totalmente de acuerdo» (Aguilar y Osma, 2014). El ACQ-R ha mostrado buena consistencia interna y validez convergente (Brown y cols., 2004; White, Brown, Somers y Barlow, 2006). Para este estudio hemos utilizado la versión traducida al castellano y validada por Barrada y cols. (2012).

\section{Procedimiento}

El ACQ-R fue completado por los pacientes como parte de la evaluación que recibieron en sus centros clínicos respectivos. En el caso de los participantes con TLP, lo completaron en el centro clínico Previ, un centro especializado en la atención de los trastornos de personalidad y trastornos de la conducta alimentaria. Respecto a los participantes con TP, la gran mayoría recibieron la evaluación en el SAP de la Universitat Jaume I, un servicio dirigido tanto a la comunidad universitaria como a personas externas de la universidad que requieran ayuda psicológica, y el resto en el centro clínico Creos, un centro de psicología clínica general. 


\section{Análisis de datos}

Para analizar los datos hemos utilizado el programa estadístico SPSS versión 20.0. Hemos empleado una comparación de medias para muestras independientes ( $T$ de student) ya que, con ello, queremos evaluar si existen diferencias significativas entre las medias de dos grupos diferentes (TP y TLP) en una misma variable dependiente (PCA).

\section{Resultados}

Para observar las diferencias en la PCA hemos comparado las medias de las dos muestras independientes a través de la prueba T. Los resultados se muestran en la tabla 1 :

Tabla 1

Comparación de medias de las diferentes subescalas del ACQ-R, ACQ-R total y edad en TP y TLP

\begin{tabular}{lrr}
\hline & \multicolumn{1}{c}{ TP } & \multicolumn{1}{c}{ TLP } \\
\hline Control emocional & $8,42(5,00)$ & $8,64(4,46)$ \\
Control del miedo & $17,86(5,9)$ & $13,64(6,95)$ \\
Control de estrés & $8,9(4,14)$ & $6,09(4,84)$ \\
ACQ-R-TOTAL & $35,28(10,94)$ & $28,5(12,6)$ \\
Edad & $32,01(10,39)$ & $26,66(8,49)$ \\
\hline
\end{tabular}

El análisis de comparación de medias muestra que el grupo de TP tiene una media de $35,02(10,94)$ en el ACQ-R total, valor superior al del grupo de TLP, con una media de 28,50 $(12,6)$. Respecto a las diferentes subescalas, observamos que en la subescala de control emocional el grupo de TP tiene una media de 8,42 (5,00) mientras que el TLP 8,64 $(4,46)$; en la subescala de control del miedo, el grupo de TP muestra un valor medio de 17,86 $(5,90)$, valor mayor al del grupo de TLP, con 13,64 $(6,95)$; por último, en la subescala de control del estrés, el grupo de TP señala una puntuación media de 8,98 $(4,14)$, valor también ligeramente superior al del grupo de TLP, con una media de 6,09 $(4,84)$. También es necesario señalar edad la media en los dos grupos, que es de $32,01(10,39)$ para TP y $26,6(8,49)$ para TLP. Para apreciar si estos resultados son superiores o inferiores a la media, los comparamos con los resultados de una muestra de trastorno de pánico clínica española. Los resultados fueron 8,31 $(4,42)$ en control emocional, $17,23(5,88)$ en control del miedo, 8,10 $(4,53)$ en control del estrés y 33,65 (10.69) en ACQ-R total (Osma y cols., en prensa). Como podemos observar, los resultados son similares en el caso TP e inferiores el TLP, de este modo podemos confirmar nuestra segunda hipótesis. 
Tabla 2

Comparación de medias en muestras independientes con prueba T

\begin{tabular}{lccc}
\hline & \multicolumn{2}{c}{ Prueba T para igualdad de medias } \\
\hline Edad & $\mathrm{t}$ & $\mathrm{gl}$ & Sig. (bilateral) \\
Control emocional & 2,895 & 103 & 0,005 \\
Control del miedo &,- 236 & 103 & 0,814 \\
Control de estrés & 3,35 & 103 & 0,001 \\
AcQ-R-TOTAL & 3,28 & 103 & 0,001 \\
\hline
\end{tabular}

Prueba de Levene para igualdad de varianzas $>0,05$

Por un lado, la prueba Levene para la igualdad de varianzas nos muestra valores superiores a 0,05 en todas las variables, por lo que podemos asumir varianzas iguales. En cuanto a la prueba T para muestras independientes, observamos que en la variable $A C Q-R$ total, $t(103)=2,940, p=0,004$ valor inferior a 0,05 , por lo que podemos afirmar que hay diferencias significativas entre el grupo TP y TLP siendo más baja en el grupo de TLP (véase la tabla 1). En la subescala de control emocional muestra que $\mathrm{t}(103)=-236, p=0,814$, valor superior a 0,05 , por lo que podemos concluir que no existen diferencias significativas entre ambos grupos. Respecto a las subescala de control del miedo apreciamos que $t(103)=3,350, p=0,001$ y en la escala de control de estrés observamos que $\mathrm{t}(103)=3,280, \mathrm{p}=0,001$, ambas con un nivel de significación inferior a 0,05 , por lo que nos permite decir que existen diferencias significativas entre el grupo TLP y TP para estas dos subescalas, con un valor inferior en el grupo TLP (véase la tabla 1). De esto modo rechazamos la primera hipótesis, en la que predecíamos unas puntuaciones parecidas de PCA en ambos grupos, ya que en todas las variables existen diferencias significativas a excepción de la subescala de control emocional, donde no ha habido diferencias significativas.

\section{Discusión}

Este estudio tiene como objetivo comparar la PCA en dos trastornos distintos, en el trastorno límite de la personalidad, que es el que nos atañe, y en el trastorno de pánico. Debido a la gran comorbilidad que existe entre ambos trastornos (McGlashan y cols., 2000; Zimmerman y Mattia, 1999; Leichsenring y cols., 2011), nuestra primera hipótesis es que ambos obtendrán puntuaciones similares de PCA y, debido a la baja eficacia en la regulación emocional presente en ambos trastornos, nuestra segunda hipótesis es que ambos grupos tendrán niveles bajos de PCA. Los resultados nos indican que ambos grupos han tenido puntuaciones bajas en la escala $A C Q-R$ total y en las diferentes subescalas (control emocional, control del estrés y control de la amenaza) comparadas con una muestra clínica española de TP (Osma y cols., prensa), por lo que se confirma nuestra segunda hipótesis. Sin embargo, es llamativo que las puntuaciones del grupo con TLP hayan sido significativamente más bajas, exceptuado en la subescala control emocional, donde han sido similares. De este modo, rechazamos nuestra primera hipótesis donde predecíamos un nivel similar de PCA entre los dos grupos. 
Que el PCA sea bajo en ambos trastornos es congruente con la literatura, donde numerosos estudios han señalado que la percepción de eventos desagradables como impredecibles e incontrolables es una característica central de la ansiedad y los trastornos del estado de ánimo (Barlow, 2002; Mineka, Watson y Clark, 1998; Zvolensky, Lejuez y Eifert, 2000).

Por otro lado, que el TLP haya dado valores significativamente más bajos puede deberse, como señalaban Chorpita y Barlow (1998), a la exposición temprana de estímulos incontrolables o impredecibles, como viene a ser haber crecido en un ambiente invalidante, donde la expresión de las emociones es rechazada por los cuidadores y sus problemas son menospreciados; consecuentemente, el niño no aprende a modular la activación emocional o a manejar el estrés (Crowell, Beauchaine y Linehan, 2009). Bray y cols. (2007) también resaltaron la dificultad que tienen los individuos con TLP a la hora de resolver problemas, sobre todo bajo situaciones de estrés; sin embargo, no se conoce demasiado sobre esta variable.

Por último, que las puntuaciones en la subescala de control emocional no sean tan bajas podría tener que ver con algunos resultados que se están obteniendo recientemente. Kuo y cols. (2016) quisieron medir la reactividad emocional y la regulación emocional volitiva en pacientes con TLP. Contrariamente a lo que se ha hipotetizado en teorías prominentes sobre el TLP (por ejemplo, Linehan, 1993; Selby y Joiner, 2009), los resultados mostraron que los individuos con TLP podían reducir su reactividad emocional a través de la regulación (siguiendo instrucciones para aplicar estrategias de regulación como el mindfulness o la distracción) tan satisfactoriamente como los sujetos del grupo control, aunque representaban una actividad basal psicofisiológica anormal. Así mismo, otros estudios han señalado que la reactividad emocional en el TLP puede que sea específica a ciertas emociones (Gratz y cols., 2010; Kuo y cols., 2013), por lo que quizá sea necesario que los estímulos provoquen las emociones clave en los individuos con TLP (Kuo y cols., 2016). De este modo, que las puntuaciones en el control emocional hayan sido bajas es coherente con esta literatura, pero el motivo por el que no hayan sido tan bajas como en el resto de subescalas puede que se deba a que los individuos con TLP tienen mayor PC a ciertas emociones que a otras.

Las limitaciones de este estudio han sido varias. Por un lado, nos hemos encontrado con varios inconvenientes con la muestra. Hay diferencias significativas con la edad de los distintos grupos, por lo que quizá que el grupo TLP haya obtenido puntuaciones significativamente más bajas en casi todas las escalas puede haber estado influido en mayor o menor medida por la variable edad. De este modo, sería conveniente reproducir la investigación con una muestra de edad equilibrada con el fin de descartar posibles interacciones. También sería mejorable para futuras investigaciones el tamaño de la muestra si queremos generalizar los resultados. Por otro lado, hemos encontrado dificultados a la hora de comparar los resultados, ya que aún no existen baremos del ACQ-R en población española. De este modo, si queremos profundizar en el estudio de la PCA, sería recomendable validar los resultados del ACQ-R en población española y obtener baremos.

Como se plantea al inicio de este estudio, conocemos muy poco sobre la influencia que ejerce la ansiedad como factor de riesgo para el desarrollo y el mantenimiento del TLP, y aún menos la PCA. Los resultados obtenidos en este estudio han sido reveladores, mostrando que el TLP tiene una considerable peor PCA que el TP, el cual sí que había sido examinado más detalladamente, por lo que animamos a las futuras líneas de investigación a profundizar sobre esta área, detallar cuáles son los factores etimológicos y mantenedores relaciones con el bajo PCA en el TLP, como por ejemplo la influencia de la dificultad para resolver problemas en la PCA, así como analizar la relación existente entre la subescala emocional del ACQ-R y la reactividad a emociones específicas. El mejor conocimiento sobre esta área podría ayudarnos a mejorar los programas de tratamiento y, en última instancia, ayudar a mejorar el bienestar de uno de los trastornos de personalidad más problemáticos. 


\section{Referencias bibliográficas}

Aguilar, A. y Osma, J. (2014). Validación Española del Cuestionario de Control de la AnsiedadRevisado $(A C Q-R)$. España: Universidad de Zaragoza.

American Psychiatric Association (1994). Diagnostic and Statistical Manual of Mental Disorders (4th edition). Washington, DC: APA.

Barrada, J. R., Osma, J., García-Palacios, A., Crespo, E., Fermoselle, E. y Castellano, C. (2012). Validación española del cuestionario de percepción de control de la ansiedadrevisado. Documento presentado en el V Congreso Internacional y X Nacional de Psicología Clínica, Santander, España.

Barlow, D. H. (2002). Anxiety and its disorders: The nature and treatment of anxiety and panic (2nd ed.). New York, NY: Guilford Press.

Barlow, D. H., Brown, T. A., Somers, T. J. y White, K. S. (2006). Avoidance behavior in panic disorder: The moderating influence of perceived control. Behaviour Research and Therapy, 44, 147-157.

Bray, S., Barrowclough, C. y Lobban, F. (2007). The social problem-solving abilities of people with borderline personality disorder. Behaviour Research and Therapy, 45, 1409-1417.

Brown, T. A., White, K. S., Forsyth, J. P. y Barlow, D. H. (2004). The structure of perceived emotional control: Psychometric properties of a revised anxiety control questionnaire. Behavior Therapy, 35, 75-99.

Chapman, L. K., Kertz, S. J. y Woodruff-Borden, J. (2009). A structural equation model analysis of perceived control and psychological distress on worry among AfricanAmerican and European American young adults. Journal of Anxiety Disorders, 23, 69-76.

Chorpita, B. F. y Barlow, D. H. (1998). The development of anxiety: The role of control in the early environment. Psychological Bulletin, 124, 3-21.

Crowell, S., Beauchaine, T y Linehan, M. (2009). A Biosocial Developmental Model of Borderline Personality: Elaborating and Extending Linehan's Theory. Psychological Bulletin, 135, 495-510.

Cox, B., Endler, N., Swinson, R. y Norton, G. (1992). Situations and specific coping strategies associated with clinical and nonclinical panic attacks. Behaviour Research and Therapy, 30, 67-69.

Ferrer, M. (2013). Trastorno Límite de la Personalidad: Diferentes manifestaciones de un mismo trastorno. (Tesis Doctoral.) Universidad Autónoma de Barcelona. España.

Gratz, K. L. y Gunderson, J. G. (2006). Preliminary data on an acceptance-based emotion regulation group intervention for deliberate self-harm among women with borderline personality disorder. Behavior Therapy, 37, 25-35.

Gratz, K. L., Rosenthal, M. Z., Tull, M. T., Lejuez, C. W. y Gunderson, J. G. (2010). An experimental investigation of emotional reactivity and delayed emotional recovery in borderline personality disorder: the role of shame. Comprehensive Psychiatry, 51, 275-285.

Gratz, K. L., Tull, M. T. y Gunderson, J. G. (2008). Preliminary data on the relationship between anxiety sensitivity and borderline personality disorder: The role of experiential avoidance. Journal of Psychiatric Research, 42, 550-559.

Gunderson, J. G. y Singer, M. T. (1975). Defining borderline patients: An overview. American Journal of Psychiatry, 132, 1-10.

Kuo, J., Fitzpatrick, S., Metcalfe, R. y McMain S. (2016). A multi-method laboratory investigation of emotional reactivity and emotion regulation abilities in borderline

Kuo, J. R. y Linehan, M. M. (2009). Disentangling emotion processes in borderline personality disorder: physiological and self-reported assessment of biological vulnerability, baseline 
intensity, and reactivity to emotionally evocative stimuli. Journal of Abnormal Psychology, 118, 531-544.

personality disorder. Journal Behavior Therapy \& Experimental Psychiatry, 50, 52-60.

Leichsenring, F., Leibing, E., Kruse, J., New, A. S. y Leweke, F. (2011). Borderline personality disorder. The Lancet, 377, 74-84.

Linehan, M. M. (1993). Cognitive-behavioral treatment of borderline personality disorder. New York, NY: Guilford Press.

Livesley W., Jang K. y Vernon P. (1998). Phenotypic and genetic structure of traits delineating personality disorder. Archives of General Psychiatry, 55, 941-948.

Mardiyono, M., Songwathana, P. y Petpichetchian, W. (2011). Concept analysis of perceived control. Nurse Media Journal of Nursing, 1, 225-243.

McGlashan, T., Grilo, C., Skodol, A., Gunderson, J., Shea, M., Morey, L., Zanarini, M. y Stout, R. (2000). The Collaborative Longitudinal Personality Disorders Study: Baseline Axis I/II and II/II diagnostic co-occurrence. Acta Psychiatra Scandavia, 102, 256-264.

Mineka, S., Watson, D. y Clark, L. A. (1998). Comorbidity of anxiety and unipolar mood disorders. Annual Review of Psychology, 49, 377-412.

Reiss, S. y McNally, R. J. (1985). Expectancy model of fear. En S. Reiss y R. R. Bootzin (eds.), Theoretical issues in behavior therapy (pp. 107-121). New York: Academic Press.

Rapee, R. M., Craske, M. G., Brown, T. A. y Barlow, D. H. (1996). Measurement of perceived control over anxitety-related events. Behavior Therapy, 27, 279-293.

Osma, J., Barrada, J. R, García-Palacios, A. y Botella, C. (en prensa). ACQ y gravedad de pánico.

Osma, J., García-Palacios, A. y Botella, C. (2014). Personalidad y trastorno de pánico: un estudio de revisión. Anales de Psicología, 30, 381-394.

Sandín, B., Chorot, P. y McNally, R. J. (2001). Anxiety sensitivity index: normative data and its differentiation from trait anxiety. Behaviour Research and Therapy, 39, 213-219.

Sandín, B., Chorot, P., Valiente, R. M., Santed, M. A. y Lostao, L. (2004). Dimensiones de la sensibilidad a la ansiedad: evidencia confirmatoria de la estructura jerárquica. Revista de Psicopatología y Psicología Clínica, 9,19-33.

Selby, E. A. y Joiner, T. E. Jr. (2009). Cascades of emotion: the emergence of borderline personality disorder from emotional and behavioral dysregulation. Review of General Psychology, 813, 219-229.

Siever, L. y Davis, K. (1991). A psychobiological perspective on the personality disorders. The American Journal of Psychiatry, 148, 1647-1658.

Skodol, A., Gunderson, J., Pfohl, B., Widiger, T., Livesley, W. y Siever, L. (2002). The borderline diagnosis I: psychopathology, comorbidity, and personality structure. Biological Psychiatry, 51, 936-950.

Snyder, S. y Pitts, W. (1988). Characterizing Anxiety in the DSM-III Borderline Personality Disorder. Journal of Personality Disorders, 2, 93-101.

Taylor, S., Koch, W. J. y Crockett, D. J. (1991). Anxiety sensitivity, trait anxiety and the anxiety disorders. Journal of Anxiety Disorders, 5, 293-311.

Taylor, S., Koch, W. J. y McNally, R. J. (1992). How does anxiety sensitivity vary across the anxiety disorders? Journal of Anxiety Disorders, 6, 249-259.

White, K. S. y Barlow, D. H. (2002). Panic disorder and agoraphobia. En D. H. Barlow (ed.), Anxiety and its disorders: the nature and treatment of anxiety and panic (pp. 328-379). London: The Guilford Press, New York.

White, K., Brown, T., Somers, T. y Barlow, D. (2006). Avoidance behavior in panic disorder: The moderating influence of perceived control. Behavior Research and Therapy, 44,147157. 
Zanarini, M. y Frankenburg, F. (1997). Pathways to the development of borderline personality disorder. Journal of Personality Disorders. 11, 93-104.

Zimmerman, M. y Mattia, J. (1999). Axis I diagnostic comorbidity and borderline personality disorder. Comprehensive Psychiatry, 40, 245-252.

Zvolensky, M., Lejuez, C. y Eifert, G. (2000). Prediction and control: operational definitions of the experimental analysis of anxiety. Behavior Research and Therapy, 38, 653-663.

Zvolensky, M. J., Bernstein, A., Marshall, E. C. y Feldner, M. T. (2006). Panic attacks, panic disorder, and agoraphobia: Associations with substance use, abuse, and dependence. Current Psychiatry Reports, 8, 279-285. 